\title{
Restoration of a worn-out dentition by removable and fixed prosthodontics - A case report
}

\author{
Ritika Bhambhani ${ }^{1, *}$, Santanu Sen Roy ${ }^{2}$ \\ Reader, ${ }^{1}$ Dept. of Prosthodontics, ${ }^{2}$ Dept. of Public Health Dentistry, ${ }^{\mathbf{1}, \mathbf{2}}$ Guru Nanak Institute of Dental Sciences and Research, \\ Kolkata, West Bengal, India
}

*Corresponding Author:

Email: ritikabhambhani@yahoo.co.uk

\begin{abstract}
Purpose: Rehabilitating the worn out dentition has a significant role in general well-being of the patient. There are endless deteriorating effects of attrited teeth and it needs to be attended at the earliest to prevent extensive treatment plan. Every full mouth rehabilitation case brings with it new challenges to be dealt with. But the approach should finally lead us to one aim that is a healthy stomatognathic system.

Case presentation: This case report discusses restoration of oral health of a 58-year old gentleman who complains of missing teeth and was observed to show loss of occlusal vertical dimension (OVD) with attrited teeth. The patient was philosophical by his attitude and well informed of his dental status. Rehabilitation involved fabrication of a maxillary cast partial denture, fixed prostheses for the lower missing teeth and composite build ups for anterior sextant where needed. The restorations included a reorganized approach to restore occlusion to group function with combination of fixed and removable prosthesis. The treatment was preceded by fabrication of a stabilization splint. The treatment results were satisfactory and follow up was continued for 6 month period with a revised emphasis on maintenance instructions.

Discussion: A full mouth rehabilitation is a challenging situation and customized in every case due to multiple needs of esthetics and function. This case was rehabilitated with a mixed approach of fixed and removable prosthodontics and with an aim of following de Van's principle of preservation of the remaining.

Conclusions: A full mouth rehabilitation needs to be well evaluated and diagnosed before any irreversible procedures. The final outcome should provide esthetics and function of the newly established stable stomatognathic balance. The patient compliance plays a very important role in success of such a treatment.
\end{abstract}

Keywords: Reorganized approach, Loss of OVD, Attrition of teeth, Rehabilitation, Cast partial denture.

\section{Introduction}

Dentistry has revolutionized in aspects of material science, restorative technology and the general approach to dental treatment. Patients can recognize alterations in their dentition and seek care not just for esthetics but also function. Teeth not only enhance the facial appearance, help in mastication but are quite significant in other functions like phonetics, bone preservation and the harmony of stomatognathic system. Hence the loss of teeth and deranged occlusion needs to be restored back to health. While restoring dentition the final aim is to restore function (mastication \& speech) along with the appearance.

The final outcome of any restorative treatment should be stable, obeying certain guidelines; the most important being the following- patients should be able to function from centric relation without occlusal interferences, TMJ should be allowed loading without interferences, centric stops between maxillary and mandibular teeth and presence of posterior disclusion while protrusion, harmony with tongue muscles and a stable occlusal scheme..$^{1-5}$

Dentition may present with a worn out condition due to multitude of reasons. These can range from congenital anomalies (Amelogenesis Imperfecta, Dentinogenesis Imperfecta), parafunction, skeletal deformities, deep bite or aging. ${ }^{3}$ A gradual wear of teeth is thought to be a normal process during lifetime of a patient. While restoring occlusion in any of the above, a stable stomatognathic system has to be aimed for. Two methods are usually indicated for these extensive restorative needs- 'conformative approach' if few extracoronal/ intracoronal restorations are to be planned without any change of occlusal vertical dimension (OVD) or the existing intercuspal relation or a 'reorganized approach' if multiple restorations are required. The latter may require surgical crown lengthening to restore OVD depending on the crown height and the interocclusal space. The Turner and Missirlian classification explains the different extents of tooth wear. ${ }^{6-8}$

Following is a case report of an individual representing gradual wear of teeth, who was restored back to a functional state with an aim of achieving above said guidelines.

\section{Case Report}

A 58- year old gentleman reported complaining of sensitivity in few posterior teeth and unhappy with spaces between upper front teeth. On examination 5 $\mathrm{mm}$ of diestema was observed with generalized attrition of teeth. A detailed history was recorded trying to ascertain causes for attrition. Parafunction and age related attrition was considered as the provisional 
diagnosis. Patient was unaware of any clenching or grinding. An interocclusal space of $5 \mathrm{~mm}$ was noted with a loss of occlusal vertical dimension (OVD).

Intraoral findings comprised of partially edentulous arches with missing-14, 15, 17, 18, 25, 26, 27, 28, 34, 36 and 46 (FDI tooth numbering system). There was generalized attrition including palatal surface of maxillary anteriors and lower incisal edges. Clinical pulpal exposures were found in relation to teeth 24, 33 and 35. Patient had been wearing a removable prosthesis for replacement of missing upper teeth and had temporary acrylic crowns in relation to 11 and 21 (both root canal treated which was asymptomatic and looked healthy). There was a full coverage over contoured metal crown in relation to 16 . Other findings included occlusal dental caries in relation to 47 and cervical abrasion on facial surface of 35 and 45 . Patient complained of sensitivity in 35 (Fig. 1, 2, 3). The needed intraoral periapical radiographs and Orthopantogram were done (Fig. 4). Patient conforms to category 1 of Turner and Missirlian classification. ${ }^{8}$ $\mathrm{He}$ did not present with any symptoms of the temporomandibular joint disorder. Oral hygiene was acceptable and patient seemed cooperative with a philosophical mental attitude (MM House's Classification). His medical history too revealed a good health; hence prognosis was considered fair. ${ }^{9}$

The treatment plan involved opting for reorganized approach to restore the worn out dentition and the modalities were discussed with the patient. A centric relation record was made, a mounting done in same relationship. The load bearing test and clench test indicated for an ideal position for stabilization splint. A diagnostic wax up was done in CR = ICP to visualize the treatment outcome (Fig. 5) and the following treatment was found to be required:

Preprosthetic phase: An oral prophylaxis, root canal treatment in 24, 33 and 35; and restorations in 45 and 47 in the needed surfaces as mentioned above.

Prosthetic phase- Stabilization splint was planned so as to allow an increase of OVD up to $3 \mathrm{~mm}$, which would be evaluated for 1-2 months. A cast partial denture for missing maxillary teeth; fixed prosthesis in relation to 33-37, 45-47 and full coverage crowns in relation 11, 21,24 and 16. The existing crowns had to be removed for the planned restoration. Composite build up to be done for lower anterior incisal edges, upper laterals and canines.

Maintenance phase: Explained to the patient for following the hygiene instructions and regular follow ups.

The treatment was performed, according to the discussed plan; Initial periodontal and restorative procedures were started. The occlusal plane was made to confirm to the Broderick's flag. Diagnostic Casts were prepared and mounted on a Hanau Wide Vue articulator using Hanau Spring Bow transfer. Lower cast was related by taking a centric relation record using Lucia jig and a stabilization splint of clear acrylic was fabricated on the above relation. This was adjusted in patient's mouth providing a group function with a change of $3 \mathrm{~mm}$ in OVD. This was to be worn for minimum 10-12 hours daily with a follow up for $11 / 2$ to 2 months. The patient was found to be comfortable with the new occlusal relation and dimension, and hence the preparations were done for fixed prosthesis on the lower arch. Provisional restorations (Self Cure Acrylic) were fabricated at the new restored VD and cemented using Protemp (Coltene Whaledent/GC) and an upper transitional partial denture (TPD) was fabricated to maintain the new OVD. Final restorations for lower arch (PFM) as discussed in the treatment plan were cemented. Diastema between 11 and 21 was reduced by PFM crowns but could not be completely corrected, to avoid over bulk of crowns, leaving a little gap between the definitive crowns, with patient's acceptance. Maxillary cast partial denture was planned and fabricated (Fig. 7-12). Lower anteriors were built up conservatively using nanocomposites (Tetraceram, Ivoclar vivadent). A guide to do so was prepared on the planned anterior guidance to achieve posterior disclusion.

Oral hygiene motivation was ensured and patient called for needed follow-ups. Patient was found to be comfortable and happy, occlusion was reevaluated, and hygiene found to be acceptable.

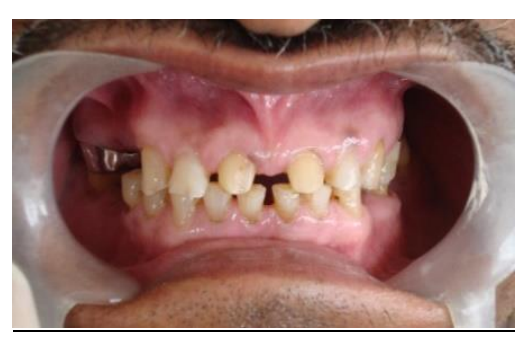

Fig. 1: Pretreatment frontal view
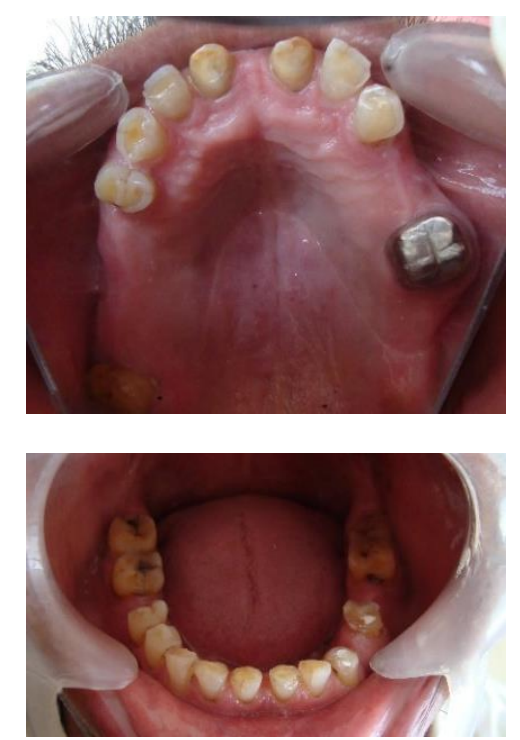

Fig. 2a and 2b: Pretreatment occlusal view 


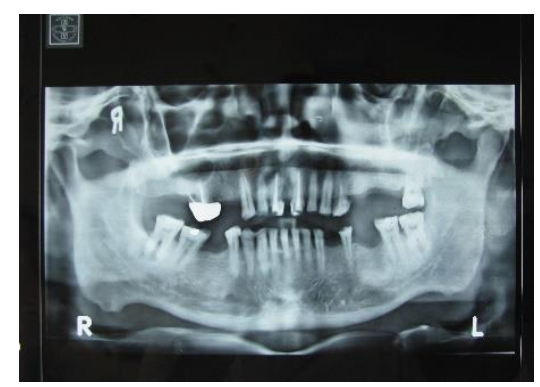

Fig. 3: OPG

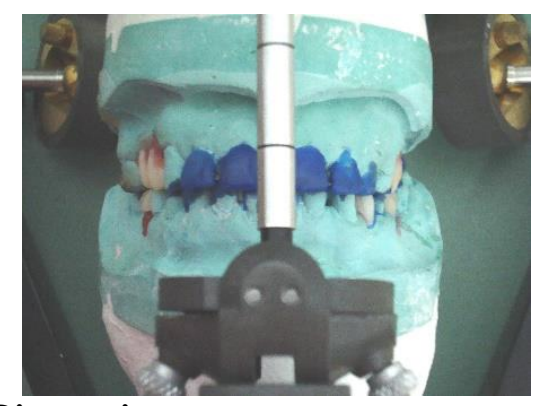

Fig. 4: Diagnostic wax up

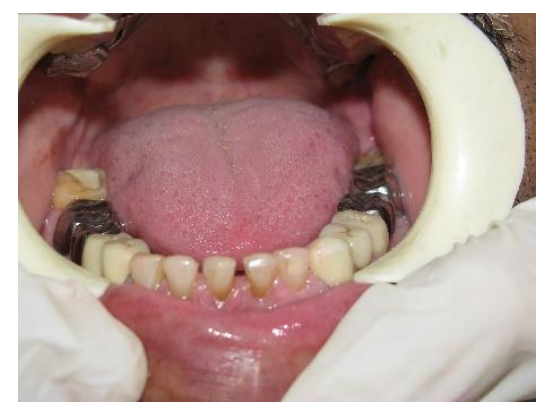

Fig. 5: Fixed prosthesis in lower arch

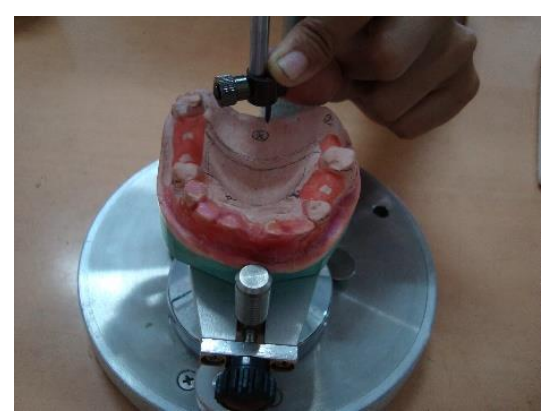

Fig. 6: Planning for CPD

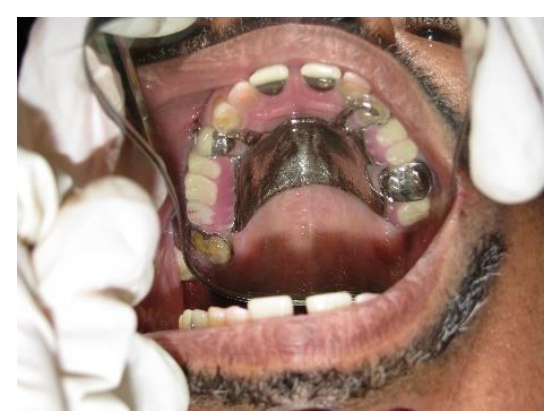

Fig. 7: After Prosthesis- Maxillary arch

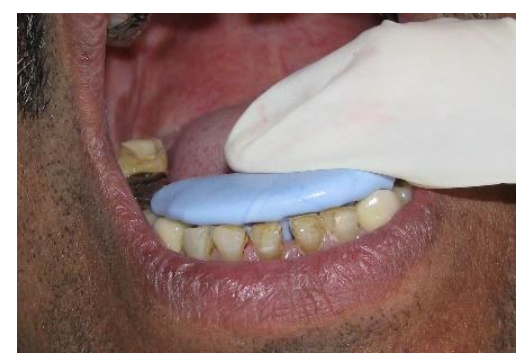

Fig. 8: Index for composite build up

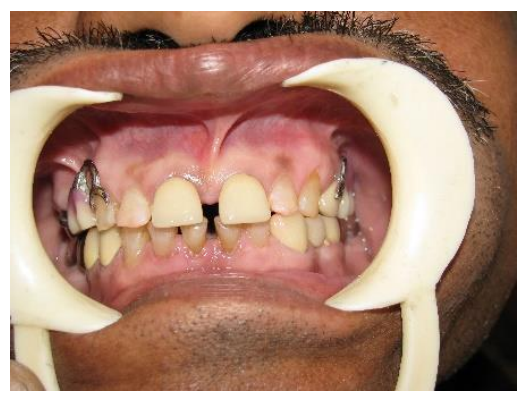

Fig. 9: Post treatment frontal view

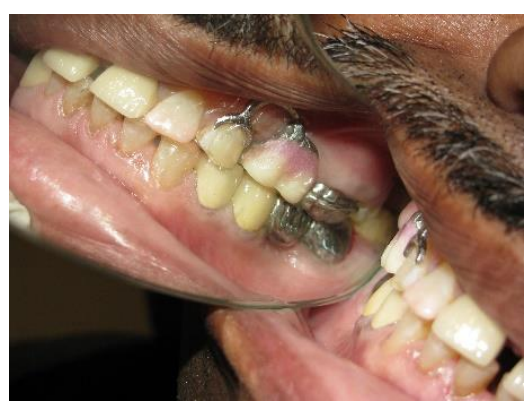

Fig. 10: Post treatment right side

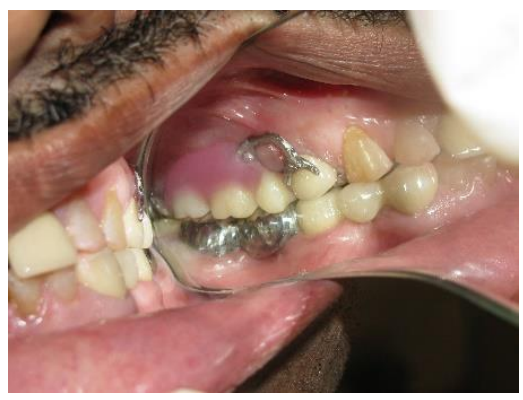

Fig. 11: Post treatment left side

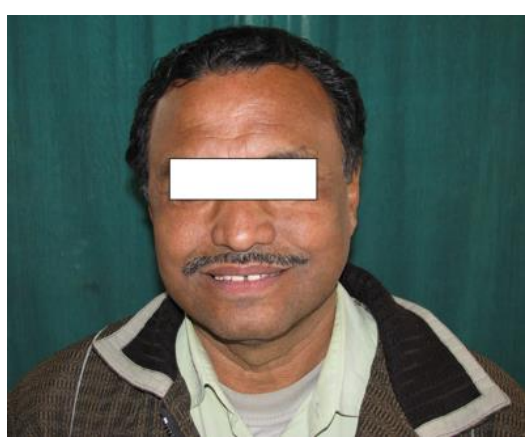

Fig. 12: Final facial view 


\section{Discussion}

Generalized attrition of teeth calls out for a restorative approach considering the periodontal, endodontic and prosthetic needs. Replacement of missing teeth follows the initial procedures and choice of treatment to be made (implants, removable partial dentures and fixed) is governed by anatomical and physiologic factors, patients' preference and economics.

Diagnosis for any case is a very significant step. In this patient a comprehensive case examination and diagnosis was done, so as not to miss important details. Any alterations which cause occlusal instability like hypermobility, tooth wear and sensitivity, broken restorations, periodontal breakdown, occlusal dimpling, exostosis, muscle enlargement, loss of posterior disclusion, multiple missing teeth, etc. may help in understanding the etiology. It has been well said "Proper planning prevents poor performance (3 P's)" Mcintre F. Taking all above considerations into effect following case has been presented. ${ }^{10-20}$

Before fabricating an irreversible fixed prosthesis it is advisable to use a reversible device such as hard maxillary occlusal splint, to evaluate patients' adaptive ability, to the new occlusal scheme, and protect the remaining tooth tissues. This was applied in the present case. Patients with natural dentition present should have treatment plan that includes periodontal management as well as prosthetic treatment for the remaining dentition within the plan for oral rehabilitation. OVD (occlusal vertical dimension) is one of the important measurements to be evaluated before starting the treatment. It not just affects the stomatognathic system functioning but also esthetics. A wide range of its values may exist without clinical symptoms although extremes of reduced vertical dimension cause fewer clinical symptoms than an OVD that is too large. Recent studies show a decrease in OVD with age without any clinical symptoms. Depending on all the evaluation pretreatment diagnostic wax-up is a must and acts like a blueprint of the final prosthesis. The existing occlusal plane should be evaluated before the fabrication of prosthesis, it is important to correct the extrusion of opposing teeth, which is often obvious on the panoramic $X$ ray film or diagnostic casts. An occlusal plane analyzer may be used on diagnostic casts to evaluate pretreatment conditions and to assist in intraoral occlusal plane correction. ${ }^{14,16}$ The occlusal plane analyzers are fabricated in several sizes; average size corresponds to 4-inch sphere and provides a starting point for ideal curves of Wilson and Spee. An acrylic shell modified with the analyzer can assist in the intraoral plane correction.

Amongst the various occlusal schemes the mutually protected occlusion is considered preferable, Gysi was one of the first to emphasize on the vertical overlap of a canine and its role in posterior disclusion. The concerned patient was preferred for the group function considering the lower vertical overlap of canines. According to Wiskott and Belser, in natural dentition, occlusal contacts are few and not ideally placed. Also functional and parafunctional forces are not directed along the long axis of the tooth. ${ }^{21}$ Based on this, they proposed a simplified occlusal scheme in which; one occlusal contact per tooth usually a cuspfossa relation is sufficient instead of a tripod contact, all interproximal contacts should be proper and tight as they stabilize the tooth mesio-distally, anterior disclusion mechanics should be applied so that posteriors do not experience any interference on lateral excursive movements, antero-posterior freedom of movement should be provided which is achieved by having concave internal slopes on the cusps of posterior teeth. A similar scheme was tried to be achieved for the concerned patient. Before beginning the treatment procedure, one must decide whether there is need for full mouth simultaneous technique which advocates simultaneous restoration of both arches, or quadrant/segment technique, where completion of restorations of one quadrant is done before proceeding to the next. This technique simplifies the essential basic procedures for reconstructions while permitting the dentist to use a suitable occlusion for a particular patient. From the belief of independent anterior guidance and condylar path, to the correlation of the two as by Hobo and Takayama concept of posterior disclusion and the Twin-tables Technique developed. The PMS philosophy combines the group function, with theory of Monson, and the functionally generated path. ${ }^{22}$ The case was to be treated by a segment approach with the final outcome to achieve group function and the lower arch first followed by the removable prosthesis of upper. The anterior sextant was rehabilitated as conservatively as possible using light cure composite build up. The developments in adhesive dentistry have proved to be a boon for conservative treatment.

\section{Conclusion}

For occlusal rehabilitation 3 factors play an important role, stable posterior contacts, stable posterior controls /TMJ and anterior control or incisal guidance (6). Any full mouth rehabilitation demands a wellexecuted treatment plan. Patients' compliance also plays a significant role in success of the treatment. The patient was rehabilitated using a conservative approach and the treatment results were good considering the post treatment follow up and patient's attitude.

Funding: No funding sources.

Conflict of interest: None declared.

\section{References}

1. Carlsson GE, Ingerval B, Kocak G. Effect of increasing vertical dimension on the masticatory system in subjects with natural teeth. J Prosthet Dent 1979;41:284-89. 
2. CS Chu F, SC Siu A, RH Newsome P, W Chow T, J Smales R. Restorative management of the worn dentition: 4. Generalized tooth wear. Dent Update 2002; 29:318-24.

3. Dawson PE. Evaluation, diagnosis and treatment of occlusal problems. $2^{\text {nd }}$ Edition, St.Louis: Mosby 1989. Pg.471

4. A systematic approach to full-mouth reconstruction of the severely worn dentition. J Lerner Pract Proced Aesthet Dent 2008;20(2):81-7.

5. Krishna MG, Rao KS, Goyal K. Prosthodontic management of severely worn dentition including review of literature related to physiology and pathology of increased vertical dimension of occlusion. J Indian Prosthod Soc 2005;5(2):89-93.

6. T-Thavornthanasarn S. Treatment of a patient with severely worn dentition: A clinical report. J Prosthodont 2007; $16: 219-25$

7. Wassel RW, Steele JG, Welsh G. Considerations when planning occlusal rehabilitation. A review of the literature. Int Dent J 1998;48;571-81.

8. Turner KA, Missirlian DM. Restoration of extremely worn dentition. J Prosthet Dent 1985;52:467-74.

9. Gamer S, Tuch R, Garcia LT. M.M.House mental classification revisited: Intersection of particular patient types and particular dentists' needs. J Prosthet Dent 2003;89(3):297-302.

10. Donegan SJ, Knap FJ. A study of anterior guidance. $J$ Prosthodont 1995;4(4):226-32.

11. Ekfeldt A, Karlsson S. Changes of masticatory movement characteristics, after prosthodontic rehabilitation, of individuals with extensive tooth wear. Int J Prosthodont 1996;9:539-46.

12. Jayachandran S, Ramachandran CR, Varghese R. Occlusal plane orientation: A statistical and clinical analysis in different clinical situations. J Prosthodont 2008; 17:572-75.

13. Mcintyre F. Restoring esthetics and anterior guidance in worn anterior teeth- A conservative multidisciplinary approach. J American Dent Assoc 2000;131:1279-83.

14. Carl E. Misch. Contemporary Implant Dent 2007. $3^{\text {rd }}$ ed.

15. Mi-Young Song, Ji Man Park, Eun-Jin Park. Full mouth rehabilitation of the patient with severely worn dentition:a case report. J Adv Prosthodont 2010;2(3):1-6110.

16. Ow RKK, Djeng SK, Ho CK. Orientation of the plane of occlusion. J Prosthet Dent 1990:64(1):31-6.

17. Schuyler $\mathrm{CH}$, Factors in occlusion applicable to restorative dentistry. J Prosthet Dent 1953;3:722.

18. Schuyler $\mathrm{CH}$. The function and importance of incisal guidance in oral rehabilitation. J Prosthet Dent 2001;86(6):219-32.

19. Tiwari B, Ladha K, Lalit A, Naik D. J Occlusal Concepts in Full Mouth Rehabilitation: An Overview. Indian Prosthodont Soc (Oct-Dec 2014) 14(4):344-351. DOI 10.1007/s13191-014-0374-y

20. Mann AW, Pankey LD. Oral rehabilitation: part I. Use of the P-M instrument in treatment planning and in restoring lower posterior teeth. J Prosthet Dent 1960;10:135-150. doi: 10.1016/0022-3913(60)90098-6.

21. Wiskott HWA, Belser UC. A rationale for a simplified occlusal design in restorative dentistry: historical review and clinical guidelines. J Prosthet Dent 1995;73:169183. doi: 10.1016/S0022-3913(05)80159-3.

22. Tiwari B, Ladha K, Lalit Aaruti, Naik BD. Occlusal concepts in full mouth rehabilitation: An Overview. $J$ Indian Prosthodont Soc 2014;14(4):344-51.

\author{
Abbreviations: \\ FDI- Federation dentaire internationale \\ ICP- Intercuspal position \\ OVD- Occlusal vertical dimension \\ RCP- Retruded contact position \\ RCT-Root canal treatment \\ TMJ- Temperomandibular joint
}

How to cite the article: Bhambhani R., Roy S. Restoration of a worn-out dentition by removable and fixed prosthodontics - A case report. Ann Prosthodont Restor Dent 2018;4(3):95-99. 